\title{
A comparison of the Berlin and Ghent nosologies and the influence of dural ectasia in the diagnosis of Marfan syndrome
}

Peter S. Rose, BS $S^{1,2}$, Howard P. Levy, MD, PhD ${ }^{1}$, Nicholas U. Ahn, MD', Paul D. Sponseller, $M D^{2}$, Trish Magyari, $M S^{1}$, Joie Davis, $M S N, C P N P^{l}$, and Clair A. Francomano, $M D^{l}$

\begin{abstract}
Purpose: To compare the Berlin and Ghent diagnostic criteria for Marfan syndrome and evaluate the utility of screening for dural ectasia in the diagnosis of Marfan syndrome. Methods: Review of clinical and radiographic data on 73 patients evaluated for Marfan syndrome at the National Institutes of Health. Results: Nineteen percent of patients diagnosed under the Berlin criteria failed to meet the Ghent standard. Dural ectasia was the second most common major diagnostic manifestation, and screening for dural ectasia established the diagnosis of Marfan syndrome in $23 \%$ of patients under the Ghent criteria. Conclusions: Some patients are appropriately excluded from the diagnosis of Marfan syndrome by the Ghent criteria. Determination of dural ectasia is valuable in the diagnosis of Marfan syndrome. Genetics in Medicine, 2000:2(5):278-282.
\end{abstract}

Key Words: Marfan syndrome, fibrillin, dural ectasia, Berlin diagnostic criteria, Ghent diagnostic criteria

Marfan syndrome is an autosomal dominant connective tissue disorder caused by mutations in the fibrillin-1 gene with a prevalence of approximately 1/10,000,1.2 However, molecular diagnosis is not generally available, mutation detection is imperfect, and not all fibrillin-1 mutations are associated with Marfan syndrome. For these reasons, Marfan syndrome is diagnosed clinically using a set of diagnostic criteria based on evaluation of family history, molecular data, and six organ systems.

The diagnostic criteria for Marfan syndrome were recently revised from the previous "Berlin" criteria ${ }^{3}$ into a stricter "Ghent" formulation.2 Both criteria evaluate family history and skeletal, ocular, cardiovascular, pulmonary, skin/ integumentary, and central nervous system manifestations. Under the Berlin criteria, patients are diagnosed based on involvement of the skeletal system and two other systems with at least one major manifestation (ectopia lentis, aortic dilation/ dissection, or dural ectasia). Patients with an affected firstdegree relative are required to have involvement of at least two other systems with one major manifestation preferred but not required. The revised Ghent formulation requires involvement of three systems with two major diagnostic manifestations. Additionally, the Ghent criteria provide for major skeletal mani-

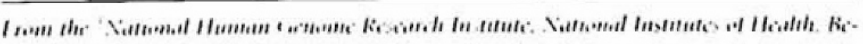

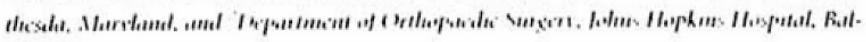
Imenere, Marrtant

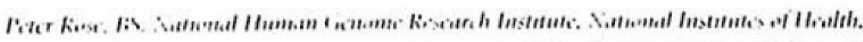

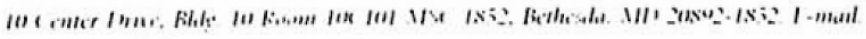

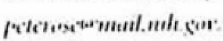

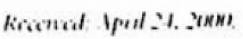

licaplical: Inte In, zaw festations and consider affected first-degree relatives or molecular data as major diagnostic criteria (Table 1).

The revision of these nosologies stemmed from concern that the older Berlin criteria did not provide for molecular data and evidence that they falsely diagnosed unaffected relatives. ${ }^{2.4 .5}$ However, some investigators have argued that the new criteria are too stringent and may exclude the diagnosis of Marfan syndrome from many affected patients. ${ }^{6}$ Similarly, the utility of screening for dural ectasia (a finding with greater impact under the revised criteria) is unknown.

The diagnosis of Marfan syndrome has important medical, personal, and reproductive consequences for patients and their families. We report a comparison of the Berlin and Ghent criteria and the influence of screening for dural ectasia in the diagnosis of Marfan syndrome among patients seen at the $\mathrm{Na}-$ tional Institutes of Health.

\section{METHODS}

We reviewed clinical and radiographic data on 73 consecutive patients evaluated for possible Marfan syndrome at the National Institutes of Health. All patients were enrolled in a molecular etiology and natural history study approved by the National Institutes of Health National Human Genome Research Institute Institutional Review Board with written informed consent (NIH protocol 97-HG-0089).

All patients were examined by at least one geneticist experienced in diagnosing Marfan syndrome and related connective tissue disorders (HPL, CAF). Aortic root dilation was defined by the criteria of Roman et al. as seen on transthoracic echocardiography. ${ }^{7}$ Dural ectasia was defined by the criteria of Ahn and colleagues based on magnetic resonance imaging (MRI) of the lumbosacral spine. ${ }^{8}$ Cornea plana was defined by keratom- 
Table 1

Berlin and Ghent diagnostic criteria

\begin{tabular}{ll}
\hline \multicolumn{2}{c}{ Berlin diagnostic criteria } \\
\hline Major involvement possible & Minor involvement possible \\
\hline Ocular system & Skeletal system \\
Cardiovascular system & Ocular system \\
Dural ectasia & Cardiovascular system \\
& Pulmonary system \\
& Skin/integumentary system \\
& Central nervous system \\
\hline
\end{tabular}

Diagnosis requires:

- In the absence of an affected $1^{\text {st }}$ degree relative, involvement of the skeleton and two other systems with at least one major manifestation.

- In the presence of an affected $1^{\text {st }}$ degree relative, involvement of at least two systems.

Ghent diagnostic criteria

\begin{tabular}{ll} 
Major involvement possible & Minor involvement possible \\
\hline Positive family history or molecular data & Skeletal system \\
Skeletal system & Ocular system \\
Ocular system & Cardiovascular system \\
Cardiovascular system & Pulmonary system \\
Dural ectasia & Skin/integumentary system \\
\hline
\end{tabular}

Diagnosis requires major involvement in two systems and minor involvement in a third.

etry measurements $<42$ diopters in adult patients (since no clear standards exist for this determination in children, we did not use this classification in pediatric patients). All patients were classified as affected or unaffected based on the published standards of the Berlin and Ghent criteria.

\section{RESULTS}

Seventy-three patients ranged in age from 1 month to 62 years at the time of evaluation. Sex ratio showed a nonsignificant male predominance ( $\mathrm{M}: \mathrm{F}=48: 25, P>0.05)$. Thirty one patients $(42 \%)$ had an affected first degree relative, and we clinically confirmed this diagnosis in 22 cases. All patients were examined by a geneticist, and all had echocardiograms, cardiac MRI imaging, and/or known aortic root dilation or dissection. Sixty-four $(88 \%)$ had complete ophthalmologic examination by ophthalmologists familiar with ocular manifestations of Marfan syndrome or known ectopia lentis. Thirty-six (48\%) had a lumbosacral MRI to evaluate dural ectasia. Complete eye examination was generally not obtained in young children. Similarly, lumbosacral MRI results were generally unavailable for claustrophobic patients, young children, and adults in whom spinal instrumentation prohibited imaging or scoliosis compromised image interpretation.
The clinical summary of patients evaluated for Marfan syndrome appears in Table 2. Nine of 48 patients (19\%) who met the Berlin diagnostic criteria did not meet the Ghent standard. This proportion did not change when only patients with complete clinical and MRI data were analyzed (seven of 32 of these patients ( $22 \%$ ) met Berlin but not Ghent diagnostic criteria). A clinical summary of the nine patients excluded under the Ghent criteria is presented in Table 3. All had either (1) no major diagnostic manifestations and an affected first degree relative or (2) aortic root dilation but no other major diagnostic manifestations.

Dural ectasia constituted the second major diagnostic manifestation (without which the diagnosis of Marfan syndrome would not be supported) for 9 of 39 patients (23\%) diagnosed under the Ghent criteria. The clinical characteristics of these patients are presented in Table 4. Presence or absence of dural ectasia had no effect on diagnoses made under the Berlin criteria. Additionally, dural ectasia was not found in any of the patients who were diagnosed under the Berlin nosology but excluded by the Ghent criteria (absent in seven, not evaluated in two).

\section{DISCUSSION}

Accurate diagnosis of Marfan syndrome has important research, medical, and personal implications for patients, but some degree of uncertainty is inherent in any diagnosis dependent on clinical criteria. For example, determination of some skeletal features is necessarily subjective, and clear guidelines do not exist for interpretation of eye findings such as keratometry measurements in children or the finding of visible zonules in the absence of ectopia lentis. Nonetheless, in the absence of a highly sensitive and specific "gold standard" for diagnosis, determination of affected status in Marfan syndrome necessarily requires the use of clinical criteria such as the Berlin or Ghent diagnostic nosologies.

These results indicate that approximately $20 \%$ of patients diagnosed with Marfan syndrome under the Berlin criteria fail to meet the Ghent diagnostic standard. Of the nine patients whose diagnoses changed under the Ghent criteria, all had either (1) no major diagnostic manifestations and an affected relative or (2) aortic root dilation but no other major diagnostic manifestations. Those in the first group all had mild systemic features often seen in the general population (for example, skin striae or mild skeletal features). Although we lack the molecular data or long-term follow-up to absolutely exclude the inheritance of a fibrillin- 1 mutation in these patients, they are clinically unaffected with Marfan syndrome. In the second group, all had aortic root dilation but no other major diagnostic features of Marfan syndrome. Three of these four patients displayed subtle systemic manifestations clinically compatible with a non-Marfan fibrillinopathy such as MASS phenotype ${ }^{9}$ or familial aortic aneurysm" rather than true Marfan syndrome. The remaining patient (Table 3 , patient number eight), a 22 year old male with a history of aortic root dilation, spontaneous pneumothorax, and minor manifestations of the skel- 
Table 2

Clinical summary of patients evaluated for Marfan syndrome

\begin{tabular}{|c|c|c|c|c|c|c|c|}
\hline & $\begin{array}{c}\text { Affected } 1^{\circ} \\
\text { relative }^{d}\end{array}$ & Skeletal & Ocular & Cardiovascular & Dural ectasia $^{d}$ & Pulmonary ${ }^{e}$ & Skin $^{e}$ \\
\hline \multicolumn{8}{|c|}{ Total $^{\prime \prime}(n=73)$} \\
\hline Involved & & $46 / 73(63 \%)$ & $17 / 64(27 \%)$ & $6 / 73(8 \%)$ & & 5 & $46 / 73(63 \%)$ \\
\hline Major & $31 / 73(42 \%)$ & $7 / 73(10 \%)^{r}$ & $12 / 64(19 \%)$ & $40 / 73(55 \%)$ & $19 / 36(53 \%)$ & & \\
\hline \multicolumn{8}{|c|}{ Berlin $^{\prime \prime}(n=48)$} \\
\hline Involved & & $44 / 48(91 \%)^{e}$ & $13 / 45(29 \%)$ & $3 / 48(6 \%)$ & & 4 & $39 / 48(81 \%)$ \\
\hline Major & $28 / 48(58 \%)$ & & $12 / 45(27 \%)$ & $38 / 48(79 \%)$ & $19 / 32(59 \%)$ & & \\
\hline \multicolumn{8}{|c|}{ Ghent $^{i}(n=39)$} \\
\hline Involved & & $34 / 39(87 \%)$ & $10 / 36(28 \%)$ & $1 / 39(3 \%)$ & & 3 & $31 / 39(79 \%)$ \\
\hline Major & $22 / 39(56 \%)$ & $5 / 39(13 \%)$ & $12 / 36(33 \%)$ & $34 / 39(87 \%)$ & $19 / 25(76 \%)$ & & \\
\hline
\end{tabular}

"All patients evaluated for possible Marfan syndrome.

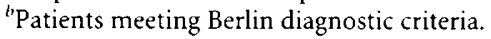

'Patients meeting Ghent diagnostic criteria.

"Major feature only; no criteria for involvement.

'Involvement only; no major criteria.

'Major involvement under Ghent standards.

etal, ocular, and skin/integumentary systems, does not meet the clinical definition of Marfan syndrome. However, he has multisystem manifestations of a Marfan-like connective tissue disorder and is clinically believed to be at risk to develop further complications consistent with the Marfan phenotype.

No case of incomplete penetrance has ever been demonstrated for patients carrying fibrillin-1 mutations associated with Marfan syndrome." However, patients with the same mutation can show a wide degree of phenotypic variability. This has been exemplified in the large pedigrees reported by Dietz and colleagues and the report of monozygotic twins with sharp differences in clinical severity of musculoskeletal and cardiovascular features of the syndrome. ${ }^{12,13}$ Continued clini- cal and molecular studies are necessary to determine the genetic risk and natural history of patients who meet the Berlin but not Ghent diagnostic criteria.

The importance of screening for dural ectasia in the diagnosis of Marfan syndrome has not been established. As a major diagnostic manifestation, the condition has greater significance under the revised Ghent criteria. Dural ectasia is most closely associated with Marfan syndrome and is a sensitive clinical manifestation of the disorder. ${ }^{14,15}$ However, dural ectasia is also found in patients with Ehlers-Danlos syndrome and neurofibromatosis, ${ }^{16}$ and the prevalence of dural ectasia in non-Marfan fibrillinopathies or other overlap connective tissue disorders has not been studied. Presence and severity of

Table 3

Clinical summary of patients meeting Berlin but not Ghent criteria

\begin{tabular}{|c|c|c|c|c|c|c|c|c|c|}
\hline Patient & Age/sex & $\begin{array}{l}\text { Family } \\
\text { history }\end{array}$ & Skeletal & Ocular & Cardiovascular & Dural ectasia & Pulmonary & Skin & Ghent score $\mathrm{e}^{d}$ \\
\hline 1 & $28 \mathrm{M}$ & Major" & Involved & No & No & No & No & Involved & $1 \mathrm{M} 2 \mathrm{I}$ \\
\hline 2 & $39 \mathrm{~F}$ & Major & Mild & No & No & No & No & Involved & $1 \mathrm{M} \mathrm{II}$ \\
\hline 3 & $16 \mathrm{~F}$ & Major ${ }^{\prime \prime}$ & Involved & No & Involved & No & No & Involved & $1 \mathrm{M} 3 \mathrm{I}$ \\
\hline 4 & $29 \mathrm{~F}$ & Major" & Mild ${ }^{c}$ & Involved & No & No & No & No & $1 \mathrm{M} \mathrm{II}$ \\
\hline 5 & $51 \mathrm{~F}$ & Major & No & No & Involved & Not eval. & No & Involved & $1 \mathrm{M} 2 \mathrm{I}$ \\
\hline 6 & $13 \mathrm{~F}$ & $\begin{array}{l}\text { Pt. } 7 \\
\text { below }\end{array}$ & No & No & Major & No & No & Involved & $1 \mathrm{M} \mathrm{II}$ \\
\hline 7 & $39 \mathrm{~F}$ & No & Mild $^{*}$ & No & Major & No & No & Involved & $1 \mathrm{M} \mathrm{II}$ \\
\hline 8 & $22 \mathrm{M}$ & No & Involved & Involved & Major & No & Involved & Involved & $1 \mathrm{M} 4 \mathrm{I}$ \\
\hline 9 & $62 \mathrm{~F}$ & No & Involved & Involved & Major & Not eval. & No & Involved & $1 \mathrm{M} 3 \mathrm{I}$ \\
\hline
\end{tabular}

"No patients meeting the Ghent diagnostic criteria failed to meet the Berlin criteria.

'We clinically confirmed the diagnosis of the affected family member.

Noted as mild if skeletal manifestations were insufficient to meet involved status under the Ghent criteria.

“Number of systems with major (M) and involved (I) status under the Ghent criteria. 
Table 4

Clinical characteristics of patients requiring presence of dural ectasia for diagnosis

\begin{tabular}{lcccc}
\hline Patient & Age/sex & Skeletal ${ }^{a}$ & Ocular & Cardiovascular \\
\hline 1 & $46 / \mathrm{F}$ & $\begin{array}{c}\text { Arachnodactyly, pectus excavatum, mild scoliosis, } \\
\text { reduced U/L segment ratio, articular } \\
\text { hypermobility, typical facies }\end{array}$ & Aortic dilation & Striae atrophica \\
\hline
\end{tabular}

$2 \quad 18 / \mathrm{F} \quad \begin{aligned} & \text { Arachnodactyly, mild pes planus, pectus } \\ & \text { excavatum, articular hypermobility, typical } \\ & \text { facies }\end{aligned}$

32/F Arachnodactyly, severe pectus excavatum, mild scoliosis, mild pes planus, reduced U/L

Striae atrophica segment ratio, articular hypermobility, typical facies

Arachnodactyly, pectus excavatum, severe pes planus, reduced U/L segment ratio and increased AS/HT ratio, articular hypermobility, typical facies

Cornea plana, increased axial length of globe
Aortic dilation

Striae atrophica

Arachnodactyly, pectus excavatum, mild scoliosis, severe pes planus, articular hypermobility, typical facies scoliosis, pes planus, increased AS/HT ratio articular hypermobility, typical facies

Striae atrophica

\section{Aortic dilation}

Aortic dilation

Striae atrophica

Aortic dilation
Recurrent herniae,
striae atrophica

${ }^{a} \mathrm{U} / \mathrm{L}$, upper to lower segment ratio; AS/HT, armspan to height ratio.

dural ectasia does not correlate with cardiovascular outcome in Marfan syndrome. ${ }^{15}$

Assessment of dural ectasia is often not performed during evaluation for Marfan syndrome because of cost, difficulties in interpreting images, and perceived lack of clinical benefit. The condition is best evaluated by lumbosacral MRI, although computed tomography $(\mathrm{CT})^{8.16}$ and plain radiographs can also be used for diagnosis (N.U. Ahn, unpublished data, 2000). Most authors report a prevalence of approximately $65 \%,{ }^{17} \mathrm{al}-$ though some investigators report figures as high as $92 \% .{ }^{15} \mathrm{Se}-$ verity seems to increase with age, supporting the hypothesis that a weakened dural sac expands from the cumulative effect of increased intrathecal pressure at the base of the spine from upright posture. Formal standards for the evaluation of MRI and $\mathrm{CT}$ images for dural ectasia have recently been published and should allow standardization of the diagnosis of dural ectasia. $^{8}$
Suitable MRI images of the lumbosacral spine were obtained on only half of our patients. Many were not obtained or were uninterpretable because of young age, claustrophobia, or spinal instrumentation or scoliosis. In those evaluated, dural ectasia was the second most predictive major diagnostic finding under both the Berlin and Ghent criteria. Dural ectasia constituted the second major diagnostic manifestation for one fourth of patients diagnosed under the Ghent criteria (without which the diagnosis of Marfan syndrome would not be supported, see Table 4 for clinical descriptions). Dural ectasia is also associated with headache, back pain, and nerve compression. ${ }^{17-19}$ These findings support the clinical and diagnostic value of screening patients with suspected Marfan syndrome for dural ectasia.

In summary, approximately $20 \%$ of patients diagnosed with Marfan syndrome under the Berlin criteria failed to meet the Ghent diagnostic standard. Determination of dural ectasia is 
valuable in the evaluation of Marfan syndrome and established the diagnosis under the Ghent criteria in one fourth of patients. The Ghent criteria appropriately exclude some patients, but further long-term follow-up or reliable molecular diagnostic techniques are necessary to establish the relative sensitivity and specificity of the Berlin and Ghent criteria as diagnostic tools.

\section{Acknowledgments}

This study was supported by an intramural research grant from the National Human Genome Research Institute of the National Institutes of Health.

\section{References}

1. Pyeritz RE, Mckisick VA. The Marfan syndrome: diagnosis and management. N Engl J Med 1979;300:772-777.

2. de Paepe A, Devereux RB, Dietz HC, Hennekam RC, Pyeritz RE. Revised diagnostic criteria for the Marfan syndrome. Am / Med Gentet 1996;62:417-426.

3. Beighton P, de Paepe A, Danks D, Finidori G, Gedde-Dahl T, Goodman R, Hall IG, Hollister DW. Horton W, Mckusick VA. International Nosology of Heritable Disorders of Connective Tissue, Berlin, 1986. Am J Met Gent 1988;29:581-594.

4. Pereira L, Levran O, Ramirez F, Lynch JR, Sykes B, Pyeritz. RE. Dietz HC. A molecular approach to the stratification of cardiovascular risk in families with Marfan's syndrome. N Engl J Med 1994:331:148-153.

5. Dietz HC, Pyeritz RE. Mutations in the human gene for fibrillin-1 (FBN1) in the Marfan syndrome and related disorders. Hum Mol Genet 1995:4 Spec No:17991809.

6. Kousseff BG. lennings IW. Ranells ID. The revised diagnostic criteria of Marfan syndrome: a clinical analysis [abstract]. Am/ Hum Genet 1999;65:A36.

7. Roman MJ, Devereux RB, Kramer-Fox R. O'Loughlin I. Two-dimensional echocar- diographic aortic root dimensions in normal children and adults. Am J Cardiol 1989;64:507-512.

8. Ahn N, Sponseller P, Ahn U, Nallamshetty L, Rose P, Buchowski J, Garrett E, Kuszyk B. Fishman E. Zinreich S. Dural ectasia in the Marfan syndrome: MR and CT findings and criteria. Genet Mcd 2000;2:186-192.

9. Dietz HC, McIntosh I, Sakai LY, Corson GM, Chalberg SC, Pyeritz RE, Francomano CA. Four novel FBNI mutations: significance for mutant transcript level and EGFlike domain calcium binding in the pathogenesis of Marfan syndrome. Genomics 1993:17:468-475.

10. Abuelo DN, Guo D, Cantu A, Carmical S, Milewicz. D. Familial aortic aneurysms [abstract]. Genet Med 2000;2:75.

11. Online Mendelian Inheritance in Man (OMIM), Johns Hopkins University, Baltimore, Maryland. Marfan syndrome, type I; MIM Number 154700: 4/24/00. World IVide IVeb URL: http://www.ncbi.nlm.nih.gov/omim/

12. Dietz HC, Pyeritz RE, Puffenberger EG, Kendzior RJ Jr, Corson GM, Maslen CL. Sakai LY, Francomano CA, Cutting GR. Marfan phenotype variability in a family segregating a missense mutation in the epidermal growth factor-like motif of the fibrillin gene. / Clin Invest 1992;89:1674-1680.

13. Ambani LM, Gelehrter TD, Sheahan DG. Variable expression of Marfan syndrome in monozygotic twins. Clin Genet 1975;8:358-363.

14. De Paepe A. Dural ectasia and the diagnosis of Marfan's syndrome. Lancet 1999:89: $878-879$.

15. Fattori R, Anienaber C, Descovich B, Ambrosetto P, Reggiani LB, Pepe G, Kaufmann U. Negrini E, von Kodolitsch Y. Gensini GF. Importance of dural ectasia in phenotypic assessment of Marfan's syndrome. Lancet 1999;354:910-913.

16. Villeirs GM, Van Tongerloo AI, Verstraete KL, Kunnen MF, De Paepe AM. Widening of the spinal canal and dural ectasia in Marfan's syndrome: assessment by CT. Neuroradiology 1999;41:850-854.

17. Pyeritz RE. Fishman EK, Bernhardt BA, Siegelman SS. Dural ectasia is a common feature of the Marfan syndrome. Am / Hum Genet 1988;43:726-732.

18. Ahn N, Sponseller P, Ahn U, Nallamshetty L, Kuszyk B, Zinreich S. Dural ectasia is associated with back pain in the Marfan syndrome. Spine 2000;25:1562-1568.

19. Stern WWE. Dural ectasia and the Marfan syndrome. I Neurosurg 1988:69:221-227. 